\title{
Telomere DNA recognition in Saccharomycotina yeast: potential lessons for the co-evolution of ssDNA and dsDNA-binding proteins and their target sites
}

\author{
Olga Steinberg-Neifach ${ }^{1,2}$ and Neal F. Lue ${ }^{1 *}$ \\ ${ }^{1}$ Department of Microbiology and Immunology, W. R. Hearst Microbiology Research Center, Weill Medical College, Cornell \\ University, New York, NY, USA, ${ }^{2}$ Hostos Community College, City University of New York, Bronx, NY, USA
}

OPEN ACCESS

Edited by:

Arthur J. Lustig,

Tulane University, USA

Reviewed by:

F. B. Johnson,

University of Pennsylvania, USA

Giovanni Cenci,

Sapienza University of Rome, Italy

*Correspondence:

Neal F. Lue,

Department of Microbiology and

Immunology, W. R. Hearst

Microbiology Research Center, Weill Medical College, Cornell University,

1300 York Avenue, New York,

NY 10065, USA

nflue@med.cornell.edu

Specialty section:

This article was submitted to

Genetics of Aging,

a section of the journal

Frontiers in Genetics

Received: 11 February 2015 Accepted: 10 April 2015

Published: 01 May 2015

Citation:

Steinberg-Neifach $O$ and Lue NF (2015) Telomere DNA recognition in

Saccharomycotina yeast: potential lessons for the co-evolution of SsDNA and dsDNA-binding proteins and their

target sites.

Front. Genet. 6:162.

doi: 10.3389/fgene.2015.00162
In principle, alterations in the telomere repeat sequence would be expected to disrupt the protective nucleoprotein complexes that confer stability to chromosome ends, and hence relatively rare events in evolution. Indeed, numerous organisms in diverse phyla share a canonical 6 bp telomere repeat unit (5'-TTAGGG-3'/5'-CCCTAA-3'), suggesting common descent from an ancestor that carries this particular repeat. All the more remarkable, then, are the extraordinarily divergent telomere sequences that populate the Saccharomycotina subphylum of budding yeast. These sequences are distinguished from the canonical telomere repeat in being long, occasionally degenerate, and frequently non-G/C-rich. Despite the divergent telomere repeat sequences, studies to date indicate that the same families of single-strand and double-strand telomere binding proteins (i.e., the Cdc13 and Rap1 families) are responsible for telomere protection in Saccharomycotina yeast. The recognition mechanisms of the protein family members therefore offer an informative paradigm for understanding the co-evolution of DNA-binding proteins and the cognate target sequences. Existing data suggest three potential, inter-related solutions to the DNA recognition problem: (i) duplication of the recognition protein and functional modification; (ii) combinatorial recognition of target site; and (iii) flexibility of the recognition surfaces of the DNA-binding proteins to adopt alternative conformations. Evidence in support of these solutions and the relevance of these solutions to other DNA-protein regulatory systems are discussed.

Keywords: telomere, telomere-binding proteins, Saccharomycotina, co-evolution of DNA and binding proteins, gene duplication, dimerization, Rap1, Cdc13

\section{Overview}

Linear eukaryotic chromosome termini are stabilized by telomeres, which are specialized nucleoprotein complexes that suppress the recognition of the ends as double strand breaks (DSBs; de Lange, 2009; O'Sullivan and Karlseder, 2010; Jain and Cooper, 2011). This stabilization is mediated by a collection of telomeric proteins that associate directly or indirectly with the repetitive telomeric DNAs and that suppress the action of checkpoint and repair proteins. The DNA component of telomeres typically comprises a duplex region of hundreds to thousands of nucleotides and a $3^{\prime}$ overhang 
of tens to hundreds of nucleotides (referred to as the G-tail because of its G-rich nucleotide composition). Both the duplex region and $3^{\prime}$ overhang are comprised of the same short repeat unit, and both are bound by sequence-specific recognition proteins, which in turn recruit other proteins crucial for telomere protection. Because proteins recruited to the duplex telomere repeats and Gtails are both required for telomere stability, the duplex/G-tail DNA structural arrangement at chromosome ends is evidently essential for telomere function. Besides telomere protection, the other major function of telomere-bound proteins is to maintain telomere DNAs. Despite their fundamental importance, telomere DNAs can experience progressive loss owing to incomplete end replication (Olovnikov, 1996), as well as drastic truncation owing to recombinational excision or replication fork collapse (Lustig, 2003; Lansdorp, 2005). To compensate for such losses, eukaryotic cells employ telomerase and the primase-pol $\alpha$ complex to extend the G-tail and the complementary C-strand of telomeres, respectively (Autexier and Lue, 2006; Blackburn and Collins, 2011; Nandakumar and Cech, 2013; Pfeiffer and Lingner, 2013; Lue et al., 2014). Not surprisingly, these telomere extension activities are subjected to elaborate control by telomere-bound proteins in order to maintain telomere lengths within a size range that is appropriate for telomere function.

A particularly prevalent telomere repeat unit, found in various fungi, plant, metazoans, and protozoa, is $5^{\prime}$-TTAGGG- $3^{\prime} / 5^{\prime}$ CCCTAA- $3^{\prime}$. In organisms with this telomere repeat unit, the duplex region is typically recognized directly by a member of the telomere repeat binding factor (TRF) protein family, whereas the $3^{\prime}$ overhang bound directly by that of the protection of telomeres 1 (POT1) protein family (Figure 1). In most mammalian cells, for example, two TRF homologs (TRF1 and TRF2) and a POT1 homolog constitute the three direct DNA-binding components

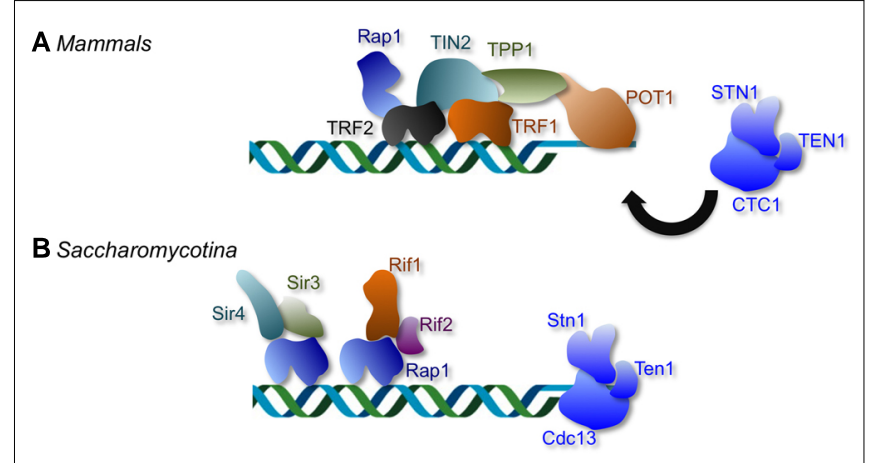

FIGURE 1 | The distinctive telomere protective complexes in mammals and in Saccharomycotina yeast. (A) The mammalian telomeres are bound by a six-protein complex collectively named shelterin. Within the telomere nucleoprotein complex, duplex telomeres are bound directly by TRF1 and TRF2, and G-tails are bound directly by POT1. The mammalian CST (CTC1-STN1-TEN1) complex plays minimal roles in telomere protection, but is crucial for regulating telomere DNA synthesis. (B) The telomere complexes of Saccharomycotina yeast display considerable differences from those in other phyla; the duplex telomeres and G-tails in Saccharomycotina yeast are bound by Rap1 and Cdc13, respectively. Like CTC1, the fungal Cdc13 is part of the CST complex that also contains Stn1 and Ten1. However, unlike mammalian CST, the fungal CST complex is crucial both for telomere protection and for regulating telomere DNA synthesis. of the six-protein "shelterin" complex that collectively protects the duplex telomeres and G-tails (Figure 1; de Lange, 2009). In fission yeast, on the other hand, a single TRF homolog (Taz1) and a POT1 homolog (Pot1) account for direct DNA-binding by a somewhat different version of the shelterin complex (Jain and Cooper, 2011). Both the TRF and POT1 family members have been subjected to extensive structural and functional investigations, and the molecular bases of their DNA recognition mechanisms are understood at the level of atomic resolution structures (Fairall et al., 2001; Lei et al., 2003, 2004; Court et al., 2005). TRF proteins form homodimers through their $\mathrm{N}$-terminal TRF homology (TRFH) domain, and use the resulting tandem Cterminal Myb DNA-binding domains (DBDs) to make contacts with two adjacent repeat units. POT1 uses a pair of OB (oligonucleotide/oligosaccharide binding) folds to interact with $\sim 10 \mathrm{nt}$ of the G-rich, $3^{\prime}$ end containing strand of telomeres [i.e., the (TTAGGG)n strand]. Sequence recognition by both proteins is highly specific such that most nucleotide substitutions in the target DNA cause a substantial loss in binding affinity. This sequence specificity is to be expected: given the capacity of the telomere proteins to "stabilize" DNA ends and prevent recombination and end-joining, promiscuous binding of these proteins to DNA DSBs would presumably be detrimental to the cells.

Implicit in the foregoing discussion are the substantial constraints imposed on the telomere nucleoprotein system during evolution. The greater constraints of the telomere system are evident when one compares its parts to those of a more circumscribed system consisting of, e.g., a transcription factor and its target site. In the latter case, a point mutation in the DNA target site could be readily accommodated by perhaps a few changes in the transcription factor DNA-binding surface. However, a comparable point mutation in the canonical telomere repeat unit is likely to cause greater disruption of cellular function and require greater compensatory adjustments. Loss of TRF or POT1 binding to the mutated repeat will probably cause extensive changes in the chromatin structure of telomeres. Conversely, restoration of normal telomere structure in this setting may require multiple changes in the binding surfaces of both TRF and POT1. Viewed in this light, it is perhaps not surprising that numerous present-day organisms in diverse phyla have retained the canonical, presumably ancient telomere repeat sequence and TRF and POT1 homologs. Examples of such organisms include fungi (e.g., basidiomycotina and pezizomycotina), metazoans (e.g., vertebrates), plants (e.g., Aloe sp., Hyacinthella dalmatica, and Othocallis siberica), and even protists (e.g., trypanosome and Leishmania), where the TTAGGG repeat is relatively uncommon (Podlevsky et al., 2008).

\section{The Unusual Telomere Repeats of Saccharomycotina Fungi}

One group of organisms with telomere systems that deviate from the canonical system is found in the Saccharomycotina subphylum of budding yeast (Figure 1). They include a widely used model organism, several pathogenic fungi, and others (Saccharomyces, Kluyveromyces, and Candida). The telomere repeats in these organisms are extraordinarily divergent and differ from the canonical repeat in being long, occasionally 
degenerate, and frequently non-G/C-rich. Notably, the telomeres of Saccharomycotina yeast are not bound directly by TRF and POT1 family members, but rather by two other distinct protein families named Rap1 and Cdc13, suggesting that the acquisition of atypical telomere DNA sequences was accompanied by a substantial remodeling of the telomere nucleoprotein structure (Figure 1). Remarkably, homologs or structural equivalents of Rap1 and $\mathrm{Cdc} 13$ also exist in organisms with the canonical telomere repeat sequence, but these homologs or equivalents clearly mediate distinct functions in these organisms. Mammalian RAP1, while a component of the shelterin complex, exhibits low affinity for telomere repeats and is localized to telomeres primarily through an interaction with TRF2 (Li et al., 2000; Arat and Griffith, 2012). The mammalian equivalent of Cdc13, named CTC1, is like Cdc13, a component of the CST (CTC1-STN1-TEN1) complex that also contains Stn1 and Ten1 (Miyake et al., 2009; Surovtseva et al., 2009). However, unlike Cdc13, CTC1 has little function in telomere protection, and appears to be primarily involved in regulating telomere DNA synthesis (Price et al., 2010; Stewart et al., 2012). The existence of mammalian CTC1 and RAP1 strongly suggests that fungal Cdc13 and Rap1 were not acquired de novo, but were co-opted to perform a new telomere function (i.e., direct telomere DNA-binding) as a pre-existing telomere component. Evolutionary models that account for the transition from the canonical telomere architecture to that found in Saccharomycotina yeast have been presented before, and will not be re-iterated in this review (Lue, 2010). Instead, we focus our discussion on a major evolutionary conundrum presented by the telomeres of this group of fungi, i.e., the DNA recognition challenge posed by rapidly evolving telomere sequence.

Interestingly, even though Rap1 exhibits little sequence similarity to TRF and has a distinct domain organization, it also utilizes Myb-like homeodomains for telomere DNA-binding. Likewise, Cdc13 can hardly be aligned to POT1 at the sequence level, yet both protein families employ the same OB fold scaffold for single-strand DNA (ssDNA) recognition. Unlike TRF and POT1, however, fungal Rap1s and Cdc13s are tasked with recognizing a very diverse collection of telomere target sequences. According to the estimates of evolutionary models, the Saccharomycotina yeasts share a common ancestor as recently as 300 million years ago, and yet collectively possess more than 20 distinct telomere repeats (Pesole et al., 1995; Hedges, 2002). A priori, this degree of evolutionary divergence can only be considered highly unusual. In terms of coding sequences, the Candida and Saccharomyces genomes are approximately as divergent as those of fish and humans, which possess the same canonical telomere sequence (Dujon et al., 2004). How then, do the major double-strand (ds) and ss telomere binding proteins (i.e., Rap1 and Cdc13) acquire the correct sequence-specificity for the rapidly changing telomere sequence? Even though we are far from having a complete answer, recent studies suggest a number of solutions to this challenge. In the following sections, we discuss in detail the structure, function and evolution of Rap1 and Cdc13, with a special emphasis on their evolutionary plasticity and their versatile DNA binding mechanisms that enables them to adapt to the multiplicity of target sequences. (In discussing the target sequence of Rap1, we will refer to the G-strand sequence such that the same strand is used in describing both the Rap1 and Cdc13 targets. This is in contrast to the majority of previous articles that characterize Rap1 binding sites.)

\section{Rap1}

Rap1 (Repressor activator protein 1, also originally known as GRF1 or TUF1), a conserved telomere protection factor, exhibit remarkable functional versatility (Shore, 1994). Notably, it was first discovered in Saccharomyces cerevisiae as a transcriptional regulator of numerous metabolic genes (Huet et al., 1985). Subsequent studies implicate Rap1 as a key component of the mating type silencer as well as the major ds telomere DNA binding protein (Shore et al., 1987; Buchman et al., 1988). That a single factor mediates such diverse functions at distinct chromosomal locations certainly raises interesting mechanistic and evolutionary issues that remain incompletely resolved. The multi-functional nature of Rap1 is evidently conserved in evolution; mammalian Rap1 has also been reported to regulate transcription and protect telomeres (Li et al., 2000; Martinez et al., 2010; Sfeir et al., 2010). However, a recent study suggests that the telomere protection function of human Rap1 may be quite minor and perhaps nonexistent (Kabir et al., 2014). At telomeres, Rap1 displays striking malleability by interacting with different molecular targets in different organisms. In budding yeast, Rap1 binds ds telomere DNAs directly with high affinity and sequence specificity, whereas in fission yeast and mammals (and probably most other organisms), Rap1 is recruited to telomeres through interaction with other telomere proteins such as TRF2 and Taz1 (Li et al., 2000; Kanoh and Ishikawa, 2001). In keeping with its multi-functional nature, $S$. cerevisiae Rap1 possesses a complex domain organization (Figure 2A). Near its $\mathrm{N}$-terminus is a BRCA1 C-terminus (BRCT) domain, a presumed protein interaction domain whose targets may include Gcr1, another transcription factor (Lopez et al., 1998). Located centrally is the DBD, which uses a pair of Myb motifs to interact with DNA (Giraldo and Rhodes, 1994; Wahlin and Cohn, 2000; Figures 2A,B). At the C-terminal end of Rap1 is a purely alpha helical structure Rap1 C-terminus (RCT) that has been shown to mediate interactions with other proteins required for proper telomere structure and function (e.g., Sir3, Sir4, Rif1, and Rif2; Feeser and Wolberger, 2008). Finally, a region between the DBD and RCT has been ascribed a transcriptional activation function (Shore, 1994). With a few exceptions (e.g., C. albicans Rap1 lacks RCT) this domain organization is conserved in other Saccharomycotina homologs. However, fission yeast and mammalian Rap1s display structural and functional differences, owing perhaps to their different means of telomere localization; these Rap1s carry a single Myb motif that binds DNA with low affinity, and an RCT that tethers Rap1 to a high-affinity DNAbinding protein (i.e., Taz1 in S. pombe and TRF2 in mammals; Li et al., 2000; Kanoh and Ishikawa, 2001; Arat and Griffith, 2012; Figures 1 and 2A).

The DNA-binding activity of the Rap1 DBD was first characterized for the $S$. cerevisiae protein, and the binding of ScRap1 to numerous DNA targets $(\sim 200-300$ promoters, two silencers, and several telomeric variants) have been investigated individually and at genome-wide scale (Idrissi and Pina, 
A

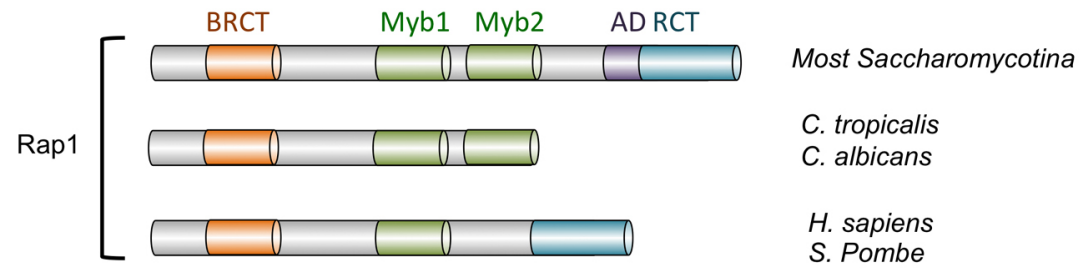

B

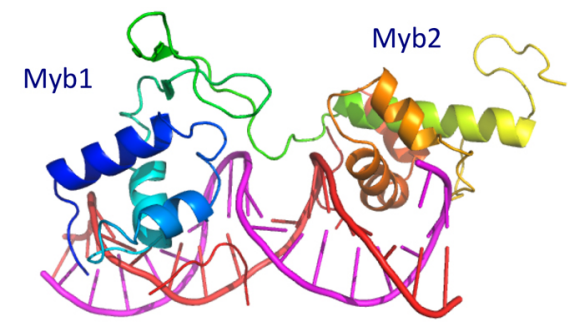

C

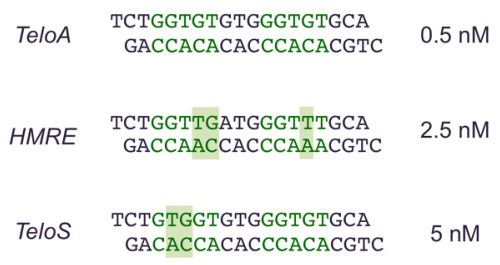

FIGURE 2 | The domain organization of Rap1 and the structure of Rap1 $1_{\text {DBD }}$-DNA complex. (A) The domain structures of Rap1 from various Saccharomycotina and other species are illustrated. The BRCT, Myb, AD (activation domain), and RCT (Rap1 C-terminal) domains are displayed in different colors. (B) The crystal structure of the Myb1 and Myb2 domains of S. cerevisiae Rap1 (shown in color spectrum from blue to orange) bound to its target DNA (shown in magenta and red; PDB ID: 1IGN). (C) The sequences of the three duplex oligonucleotides bound by ScRap1 DBD $_{\text {in a crystallographic }}$ study are displayed. The half sites in each oligo are shown in green, and nucleotides that deviate from a canonical half site $\left(5^{\prime}\right.$-GGTGT-3 $3^{\prime} / 5^{\prime}$-ACACC-3 $\left.3^{\prime}\right)$ are shown with a shaded background. The affinities of Rap $1_{\mathrm{DBD}}$ for each sequence are shown on the right. Other variant targets (e.g., the site upstream of ribosomal protein genes: AAATGTATGGGTGT) have been reported to have comparable affinities (Idrissi et al., 1998; Idrissi and Pina, 1999).
1999; Lieb et al., 2001; Pina et al., 2003; Yarragudi et al., 2007; Rhee and Pugh, 2011). While several consensus sequences for Rap1 have been reported, a frequently noted version is $\mathrm{K}_{13^{\prime}} \mathrm{R}_{12^{\prime}} \mathrm{T}_{11^{\prime}} \mathrm{G}_{10^{\prime}} \mathrm{T}_{9^{\prime}} \mathrm{R}_{8^{\prime}} \mathrm{Y}_{7^{\prime}} \mathrm{G}_{6^{\prime}} \mathrm{G}_{5^{\prime}} \mathrm{G}_{4^{\prime}} \mathrm{T}_{3^{\prime}} \mathrm{G}_{2^{\prime}} \mathrm{T}_{1^{\prime}}$ (Lieb et al., 2001). This somewhat degenerate consensus consists of two half sites, $\mathrm{K}_{13^{\prime}} \mathrm{R}_{12^{\prime}} \mathrm{T}_{11^{\prime}} \mathrm{G}_{10^{\prime}} \mathrm{T}_{9^{\prime}}$ and $\mathrm{G}_{5^{\prime}} \mathrm{G}_{4^{\prime}} \mathrm{T}_{3^{\prime}} \mathrm{G}_{2^{\prime}} \mathrm{T}_{1^{\prime}}$, bound respectively by the second and first Myb motif in Rap1. Subsets of Rap1 targets (e.g., at ribosomal protein gene promoters) exhibit distinctive features with regard to their sequences and dispositions, suggesting that the activities of Rap 1 at different chromosomal locations may be modulated by its binding to specific variants of the consensus, i.e., Rap1 may adopt different conformations, and hence recruit different co-factors depending on the specific target sequence to which it is bound (Pina et al., 2003).

As implied from the foregoing discussion, $S c R a p 1$ displays considerable flexibility in recognizing diverse target site sequences. This flexibility stems in part from the ability of the Myb motifs to tolerate many variations in the target sequence (especially the half site comprised of residues $13^{\prime}-9^{\prime}$ ) without suffering a loss in binding affinity (Vignais et al., 1990; Idrissi and Pina, 1999). This is evident from the loose consensus reported for Rap1, and especially the more degenerate sequence reported for the first half site. The molecular basis for the flexibility of Rap1 has been investigated through crystallographic analysis of three complexes formed between $S c R a p 1_{\mathrm{DBD}}$ and different DNA target sites (Figure 2C; Konig et al., 1996; Taylor et al., 2000). Overall, the results indicate that recognition of base pairs that vary between the target sites is accomplished through the utilization of alternative side-chain conformations and alternative contacts to the nucleotides. In other words, rather than altering its overall configuration, Rap1 modifies its fine surface structure to suit the demand of a particular target sequence. This inherent versatility is not unique to Rap1 (see, e.g., Schwabe et al., 1995), but appears to be highly developed in this protein, and may have allowed it to handle the challenge presented by the rapidly evolving telomere sequence in Saccharomycotina yeast (see below).

Another (probably minor) source of flexibility may be the number of nucleotides that separate the two half sites. In the vast majority of well-characterized target sites, this number is three such that the center-to-center distance between the two half sites is 8 bp (Pina et al., 2003). However, in a footprinting analysis utilizing a variant telomere sequence derived from $S$. castellii, $S c$ Rap1 produced a split footprint indicative of a center-to-center distance of $14 \mathrm{nt}$, suggesting that an atypical separation between the half sites can be tolerated in rare cases, possibly through looping out of the intervening DNA (Wahlin and Cohn, 2000).

Because all Saccharomycotina Rap1 homologs possess duplicated Myb motifs, it seems likely they all use such motif pairs for direct DNA-binding. This proposition is consistent with studies of two Rap1 family members, namely those in S. castellii and C. albicans. Specifically, the pairs of Myb motifs in each protein alone have been shown to be just as active in DNA-binding as the respective full-length protein (Wahlin and Cohn, 2002; Yu et al., 2010; Rhodin Edso et al., 2011). While not as well characterized as ScRap1, the DNA-binding mechanisms of ScasRap1 and CaRap1 also appear to be quite similar to that for $S c$ Rap 1 with respect to 

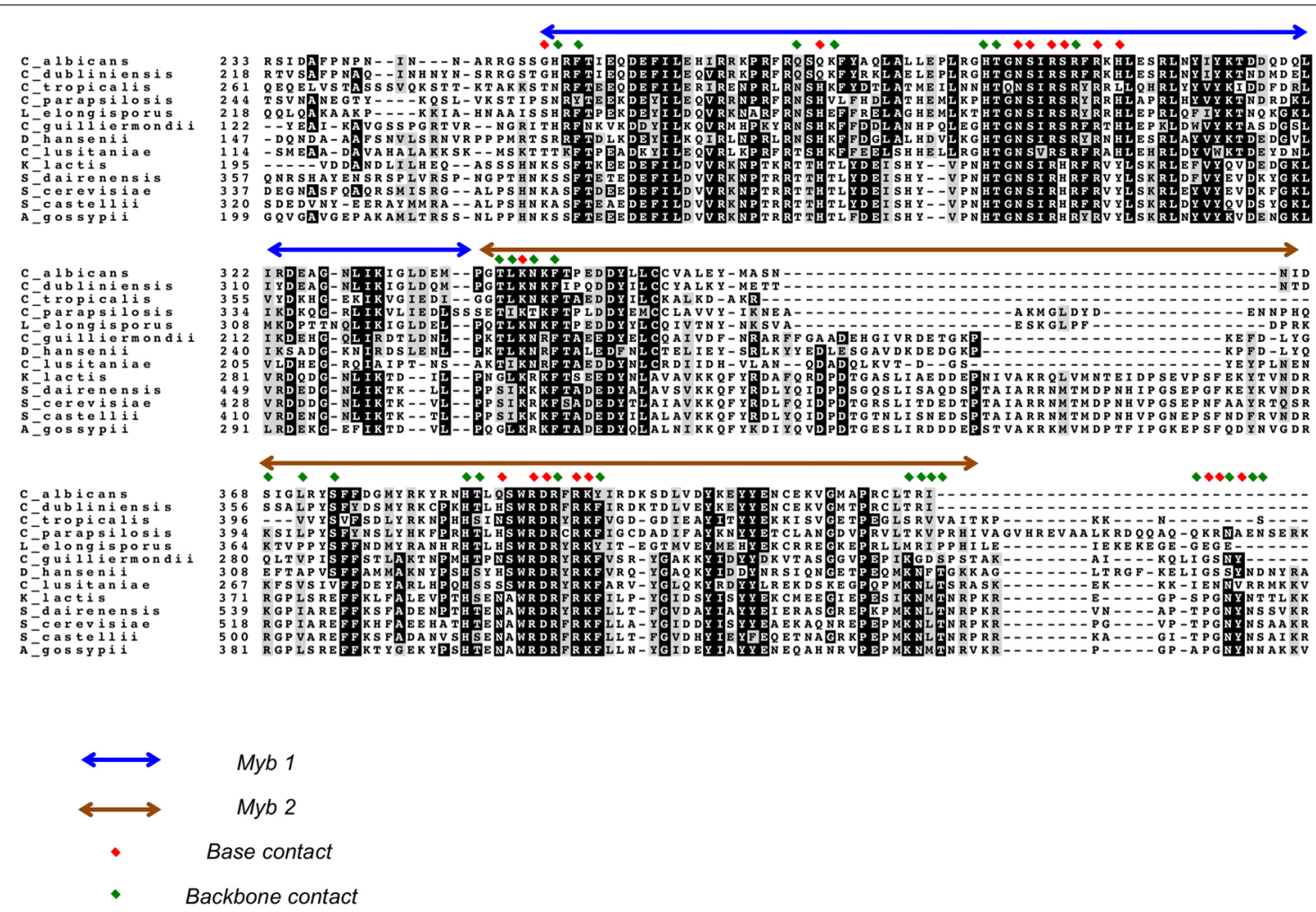

FIGURE 3 | An alignment of Rap1DBDs showing the variable extent of sequence conservation of residues implicated in DNA contacts. An alignment of the DBDs of selected Saccharomycotina Rap1 homologs is displayed. The first and second Myb motifs are indicated by blue and brown arrows, respectively. Residues implicated in making base and backbone contacts are designated by red and green diamonds, respectively. Dark and gray shading of amino acids are used to highlight strict conservation and conservative substitution, respectively. target site arrangement and sequence. For ScasRap1, the minimal high affinity target is a 12-bp duplex (GGGTGTCTGGGT), within which just three positions (G1, C7, T12) appear to have nonstringent sequence requirement (Rhodin Edso et al., 2011). For $\mathrm{CaRap1}$, the high affinity target consists of two 5-bp elements (GGTGT and GGATG) separated by two base pairs of random nucleotides (Yu et al., 2010). These observations are quite consistent with the notion of consecutive Myb motifs each recognizing 4-5 bp of G-rich elements. The exact identity of the first half site (GGTGT), which is the target of the second Myb motif according to the $S c$ Rap $1_{\mathrm{DBD}}$-DNA crystal structure, suggests that the mechanisms of this second Myb motif in telomere DNA-binding may be quite well conserved in evolution. On the other hand, the halfsite separations for ScasRap1 and CaRap1 appear to be smaller than, and the consensus sequences for their second half sites quite different from that of ScRap1, consistent with significant adaptation of these Rap1 orthologs to their cognate telomere sequences. Notably, residues in the first Myb motif of ScRap1 implicated in direct base contact appear to exhibit greater sequence variation among all the Saccharomycotina homologs than comparable residues in the second Myb motif (Figure 3). This difference could reflect adaption of the first Myb to the more divergent target sites (i.e., the second half site). A notable difference between Candida and Saccharomyces Rap1s is that the former has a far less significant role in transcriptional regulation and does not appear to bind to the promoters of many metabolism-related genes
(Lavoie et al., 2010; Yu et al., 2010). Hence, it is unclear if CaRap1 possesses the same degree of target site recognition versatility as that possessed by ScRap1. Nevertheless, the versatility exhibited by $S c$ Rap 1 indicates that members of this protein family has a variety of means to bind alternative sequences, and hence is well positioned to handle the challenge posed by the rapidly evolving telomere sequence in Saccharomycotina yeast.

\section{Cdc13}

Cdc13 (cell division cycle 13), the major G-tail binding protein in Saccharomycotina yeast, is like Rap1, a multifunctional protein with a complex domain organization (for reviews, see GiraudPanis et al., 2010; Lue, 2010). As the name implies, it was initially characterized as a gene in S. cerevisiae that when mutated, causes cell cycle defects (Garvik et al., 1995). Subsequent studies uncovered not only the G-tail binding activity of $S c \mathrm{Cdc} 13$, but also multiple functions for this protein at telomeres, including protecting telomeres against C-strand degradation, as well as regulation of both telomerase and Pol $\alpha$ in their telomere DNA synthesis activities (Nugent et al., 1996; Qi and Zakian, 2000; Pennock et al., 2001). For a subset of these functions, $S c C d c 13$ works as part of a complex (CST) that also contains Stn1 and Ten1 (Giraud-Panis et al., 2010).

Structurally, $S c \mathrm{Cdc} 13$ is quite large (924 aa) and complex, and is comprised of four $\mathrm{OB}$ fold domains that bind distinct 
A
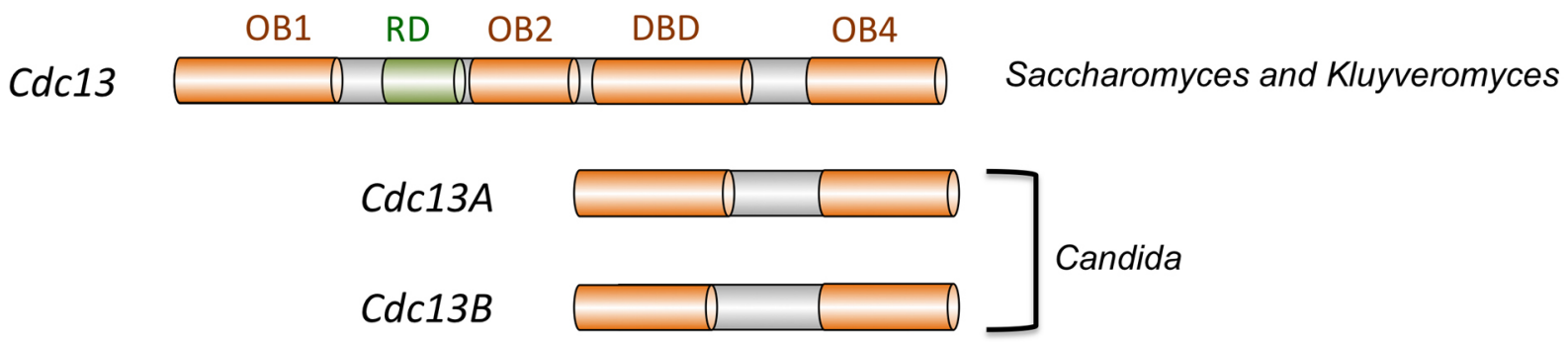

B
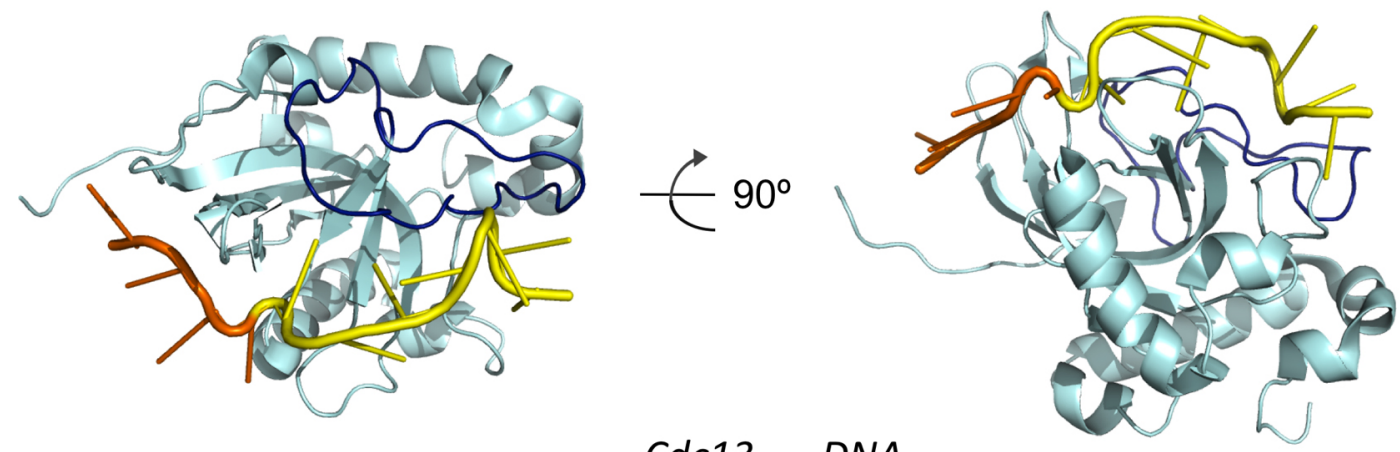

$C d c 13_{D B D}-D N A$

FIGURE 4 | The domain organization of $\mathrm{Cdc13}$ and the structure of Cdc13 ${ }_{\text {DBD }}$-DNA complex. (A) The domain structures of Cdc13 homologs from various Saccharomycotina species are illustrated. The four OB folds (OB1, OB2, $\mathrm{DBD}$, and $\mathrm{OB} 4)$ are displayed in orange, and the $\mathrm{RD}$ (recruitment domain) is shown in green. (B) The structure of the DBD of S. cerevisiae Cdc13 bound to its target DNA (PDB ID: 1S40). Cdc13 is shown in cyan with the L23 loop highlighted in dark blue. The $5^{\prime}$ four nucleotides of the DNA target is shown in orange and the $3^{\prime}$ seven nucleotides shown in yellow. molecular targets to mediate telomere protection and maintenance (Figure 4A). $\mathrm{ScCdc1} 3_{\mathrm{OB} 1}$ forms dimers to create a binding groove for Poll (the catalytic subunit of DNA polymerase $\alpha$ ), and may possess a low affinity G-strand-binding activity as well as binding sites for other proteins (Hsu et al., 2004; Mitchell et al., 2010; Sun et al., 2011). ScCdc13 $3_{\mathrm{OB} 2}$ also forms dimers and modulates interaction between Cdc13 and Stn1 (Mason et al., 2012). The third $\mathrm{OB}$ fold $\left(\mathrm{ScCdc} 13_{\mathrm{DBD}}\right)$ constitutes the high affinity $\mathrm{G}$-strand-binding domain, and the final $\mathrm{OB}$ fold $\left(\mathrm{ScCdc13} 3_{\mathrm{OB} 4}\right)$ mediates interaction with Stn1 (Hughes et al., 2000; Sun et al., 2011; Yu et al., 2012). In addition to these OB fold domains, Cdc13 also carries a telomerase recruitment domain (RD) that binds to the telomerase regulatory subunit Est1 and that is required for telomere localization of telomerase (Pennock et al., 2001; Wu and Zakian, 2011).

Interestingly, analysis of other Cdc13s in Saccharomycotina yeast revealed a high degree of structural malleability and evolutionary plasticity. While all Saccharomyces and Kluyveromyces spp. carry just one $\mathrm{Cdc} 13$ homolog that resembles structurally ScCdc13, most Candida spp. carry two Cdc13 homologs (named Cdc13A and $\mathrm{Cdc13B}$ ), each containing just two OB fold domains that align well to $S c \mathrm{Cdc} 13_{\mathrm{DBD}}$ and $S c \mathrm{Cdc} 13_{\mathrm{OB} 4}$ (Figure $4 \mathbf{A}$ ). The accumulated structural and functional evidence suggests that ScCdc13 (and other large Cdc13s) may arise through a fusion of
Cdc13A and Cdc13B in the common ancestor of Saccharomycotina yeast (Lue and Chan, 2013).

The G-tail binding activity of $\mathrm{Cdc} 13$ was first characterized (not surprisingly) for the $S$. cerevisiae protein. Proteolytic and deletion analyses defined a stable domain $\left(\mathrm{ScCdc} 13_{\mathrm{DBD}}\right.$, amino acid 557 to 692) that exhibits high affinity (sub nanomolar) for a variety of target sites that correspond to different variants of the irregular Sc G-strand repeats $\left(\mathrm{G}_{1-3} \mathrm{~T}\right.$; Hughes et al., 2000; Anderson et al., 2003). Even though full length $\mathrm{ScCdc13}$ is naturally dimeric, the DBD domain behaves as a monomer in solution and binds DNA as a monomer. The minimal size for high affinity ligands is reported to be $\sim 11 \mathrm{nt}$, and the affinities of $S c C d c 13_{\mathrm{DBD}}$ for these ligands are typically similar to or better than those of full length $S c C d c 13$. Nuclear magnetic resonance (NMR) investigations of $S c C d c 13_{\mathrm{DBD}}$ revealed an $\mathrm{OB}$ fold structure, which is quite common for ss nucleic acid-binding proteins (MittonFry et al., 2002, 2004; Figure 4B). A structural motif shared by numerous proteins, the $\mathrm{OB}$ fold is comprised of five beta strands (S1 through S5) that adopt the shape of a miniaturized barrel (Theobald et al., 2003; Bochkarev and Bochkareva, 2004). For most ssDNA-binding OB folds, residues in L12 (the loop connecting S1 and S2), L45 and the central beta strand (S3) are typically responsible for contacting a short (4-6 nt) ligand. A standard polarity prevails in the vast majority of $\mathrm{OB}$-ssDNA complexes 
such that L45 and L12 interact with the $5^{\prime}$ and $3^{\prime}$ portion of the target site, respectively. A distinctive feature of $S c \mathrm{Cdc} 13_{\mathrm{DBD}}$ is the presence of an extended and structurally well-defined L23 that makes contacts to nucleotides $3^{\prime}$ to the typical target site, thus expanding the ssDNA ligand to 11 nt (Mitton-Fry et al., 2004; Eldridge and Wuttke, 2008; Figure 4B). A combination of structural, biophysical and biochemical investigations have provided rich insights on the recognition mechanism of $S c \mathrm{Cdc} 13_{\mathrm{DBD}}$ for an 11-nt high affinity ligand (GTGTGGGTGTG; $\mathrm{K}_{\mathrm{d}}=3 \mathrm{pM}$; Anderson et al., 2003; Mitton-Fry et al., 2004; Eldridge et al., 2006; Eldridge and Wuttke, 2008). Like many other ssDNA and RNA-binding proteins, the hydrophobic and aromatic residues in $S c C d c 13_{\text {DBD }}$ evidently make greater contribution to affinity than charged residues (Anderson et al., 2003). While amino acids that contribute significantly to binding can be identified throughout the DNA-protein interface, the most critical ones all interact primarily with the $5^{\prime}$-most four nucleotides (GTGT; Anderson et al., 2003; Mitton-Fry et al., 2004). The region surrounding the $5^{\prime}$ nucleotides appear to undergo conformational re-structuring upon DNA-binding, arguing for an induced fit mechanism that may enhance the specificity of interaction (Eldridge and Wuttke, 2008). In contrast, the $3^{\prime}$ nucleotides are bound chiefly by the extended L23 with less sequence specificity, which may allow $S c C d c 13_{\text {DBD }}$ to interact optimally with the heterogeneous $S$. cerevisiae telomere repeats (Eldridge and Wuttke, 2008).

In addition to $\mathrm{ScCdc13}$, several other family members in the Saccharomyces and Candida lineages have been investigated with respect to their DNA-binding properties, revealing interesting mechanistic variations in the recognition of G-tails. ScasCdc13 is comparable in size to $S c \mathrm{Cdc} 13$, but possesses a functional DBD domain that is more extended on the N-terminal side by $\sim 70$ aa (Rhodin Edso et al., 2008). The structural basis for this additional requirement is not understood. Although the affinity of ScasCdc13 for the cognate G-tail has not been determined quantitatively, the DBD domain appears to possess an affinity similar to that of the full length protein (Rhodin Edso et al., 2008). The 8 bp minimal target site (GTGTCTGG) is somewhat smaller than the $11 \mathrm{nt}$ target site for $\mathrm{ScCdc} 13$, and the most critical nucleotide residues (positions 3, 4, 7, and 8) do not cluster near the $5^{\prime}$ end, suggesting substantial differences in the mechanism of binding (even though the GT-rich nature of the target site is conserved).

As described earlier, instead of carrying a large, 4-OB Cdc13, each Candida spp. possesses two Cdc13 homologs (Cdc13A and Cdc13B), both of which contain just 2 OB folds. Despite their small size, the Candida Cdc13s are clearly orthologs of the large 4-OB Cdc13s. Like the 4-OB Cdc13s, the Candida homologs are enriched at telomeres, and are required for normal telomere structure and function (Lue and Chan, 2013). Sequence alignments suggest that the small $\mathrm{Cdc13}$ s are structurally similar to the Cterminal half of the large $\mathrm{Cdc13}$ s, i.e., they consist of just the DBD and OB4 domains. In addition to the size difference, the small $\mathrm{Cdc13s}$ also exhibit distinct dimerization properties; whereas the large $\mathrm{Cdc13s}$ utilize OB1 for stable dimerization, the small Cdc13s appear to use primarily OB4 for this purpose (Yu et al., 2012; Lue and Chan, 2013). Moreover, in the two species for which

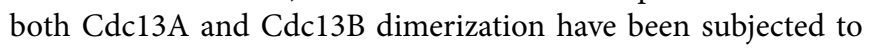

detailed analysis, the two paralogs appear to form preferentially heterodimers rather than homodimers (Lue and Chan, 2013; Steinberg-Neifach et al., 2015). Perhaps most interestingly, unlike ScCdc13, which uses a DBD monomer to mediate high affinity binding to G-tails, the Candida $\mathrm{Cdc} 13$ s evidently require protein dimerization to achieve high affinity binding (Yu et al., 2012; Lue and Chan, 2013; Steinberg-Neifach et al., 2015).

The first Candida Cdc13 complex to be subjected to detailed DNA-binding analysis is the C. tropicalis Cdc13AA homodimer (Yu et al., 2012). (This analysis was performed prior to the discovery of $C t C d c 13 B$, and the activities of the $C t C d c 13 \mathrm{AB}$ and $\mathrm{BB}$ dimer, if any, remain uncharacterized.) Investigation

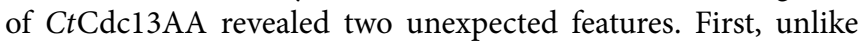
both $S c C d c 13$ and ScasCdc13, the DBD domain of CtCdc13A alone is incapable of high affinity binding to the cognate Gtail. Instead, the formation of a stable DNA-protein complex requires dimerization of full length $C t \mathrm{Cdc} 13 \mathrm{~A}$ mediated by the OB4 domain (Yu et al., 2012). Second, in keeping with the dimerization requirement, the high affinity DNA ligand consists of two copies of a 6-nt element (GGATGT) found within the $C$. tropicalis G-strand repeat unit. In the native $C t$ G-tail, the 6-nt elements are separated from one another by 17-nt, resulting in a minimal high affinity ligand (29-nt) that is far longer than those for $S c \mathrm{Cdc} 13$ and $S c a s \mathrm{Cdc13}$. Additional characterization revealed substantial spatial flexibility between the two 6-nt elements in the high affinity complex: the distance can be as short as $10 \mathrm{nt}$ (Yu et al., 2012). Thus, the individual DBDs of CtCdc13A evidently possess low affinity for a short ligand within the telomere repeat unit, requiring a pair of protein-ligand interactions conferred by the full length protein dimer to achieve high affinity Binding to G-tails.

As noted before, emerging data suggest that the Candida Cdc13s may exist preferentially as heterodimers, thus begging the question as to the recognition mechanism of this dimeric complex. This was first assessed in Candida albicans (Lue and Chan, 2013). Analysis of the C. albicans homodimers and heterodimers revealed substantial G-tail binding activities for both the AA and AB complex, but not the BB complex (Lue and Chan, 2013). However, the ligand requirements for $\mathrm{CaCdc} 13 \mathrm{AA}$ and $\mathrm{AB}$ were not examine in detail due to the propensity of these complexes to form large aggregates. The second Cdc13 heterodimer to be analyzed was from C. parapsilosis (Steinberg-Neifach et al., 2015). Similar to C. albicans, the Cdc13 paralogs in C. parapsilosis can form homo-oligomeric complexes as well as heterodimers. Surprisingly, only the $C p \mathrm{Cdc} 13 \mathrm{AB}$ heterodimer exhibits robust $\mathrm{G}$-tail binding activity. In contrast to $C t \mathrm{Cdc} 13 \mathrm{AA}$, the formation of high affinity CpCdc13AB-DNA complex requires just one copy of the 6-nt consensus element. Additional studies revealed a minimal target site of $\sim 17 \mathrm{nt}$ comprised of the 6-nt element and $11 \mathrm{nt}$ on the immediate $5^{\prime}$ side of the element. Detailed investigation of the sequence specificity coupled with site-specific crosslinking assays uncovered an unprecedented "combinatorial" mechanism of G-tail recognition. In this mode of recognition, the DBDs of $C p C d c 13 \mathrm{~A}$ and $C p \mathrm{Cdc} 13 \mathrm{~B}$ make contacts to the $3^{\prime}$ and $5^{\prime}$ region of the repeat unit, respectively. Recognitions of both regions of the repeat are highly sequence-specific, thus enabling $C p \mathrm{Cdc} 13 \mathrm{AB}$ to bind its cognate target with much greater species-specificity 
than the $C t \mathrm{Cdc} 13 \mathrm{AA}$ complex. In addition, the OB4 domains of $C p \mathrm{Cdc13A}$ and $C p \mathrm{Cdc} 13 \mathrm{~B}$ contribute to high affinity binding by forming a stable heterodimer to promote the dimerization of the DBDs. These results indicate that in some Candida spp., the challenge of binding variant G-tails is met through the duplication of $\mathrm{Cdc13}$, the hetero-dimerization of the paralogs, and the adaption of the DBDs to new target sequences. Studies of other additional Candida Cdc13s should provide insights on the general applicability of this proposal.

\section{Shared and Distinctive Features of ds and ss Telomere DNA Recognition in Saccharomycotina Yeast}

As noted before, a unique attribute of the telomere system from the evolutionary standpoint is the need to maintain adequate recognition of the telomere DNA in both its double-stranded and single-stranded forms upon changes in the sequence of the DNA. The remarkable divergence of telomere repeat sequences in Saccharomycotina yeast indicates that the Rap1 and Cdc13 protein families are sufficiently versatile and malleable to meet the challenge. While the mechanisms used by each family for DNA recognition are clearly distinct, some general themes can nevertheless be discerned. Below I list common and distinctive strategies utilized by these protein families to enable recognition of diverse sequence targets by family members.

First, the utilization of a pair of DBDs, either as parts of the same polypeptide or a dimeric complex, is probably advantageous (Figure 5). A domain with a short DNA target site may be capable of forming only a low affinity complex; incorporating two low affinity interactions in a single complex can substantially increase

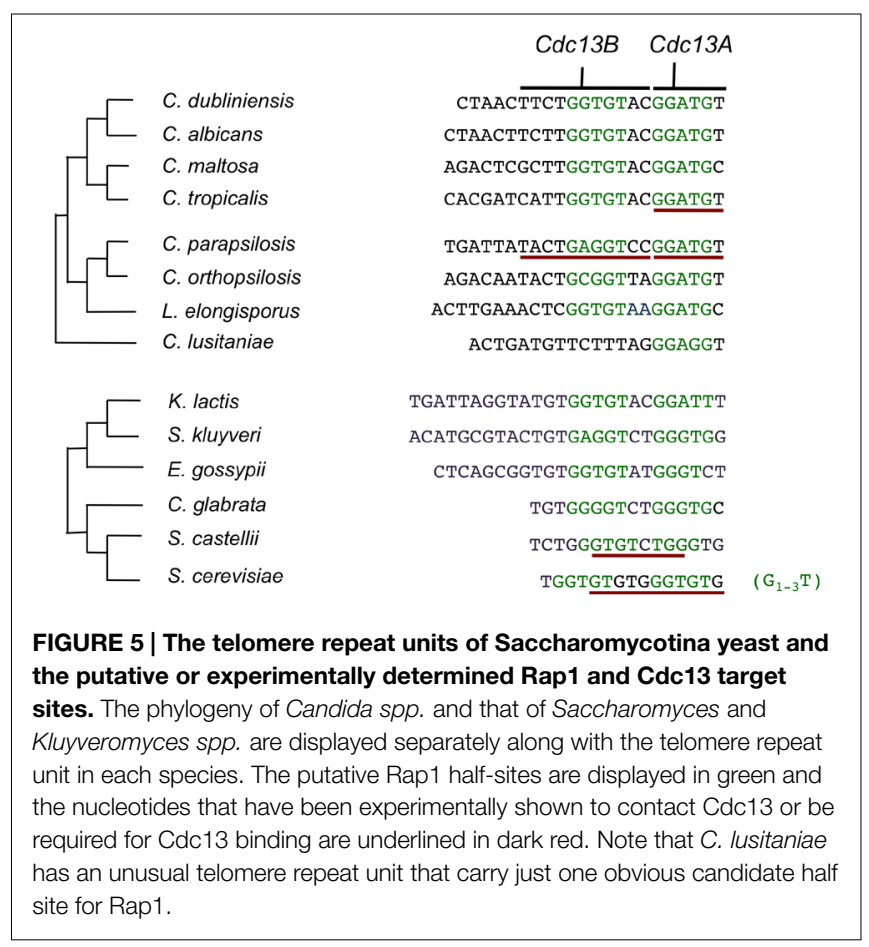

the overall affinity. The two-domain arrangement can also offer added flexibility to the system: variations in the spacing between the "half sites" are readily accommodated by two DBDs that can be flexibly positioned to each other. As illustrations, one can point to Rap1s in Saccharomycotina yeast, which have two Myb motifs and bind DNA with high affinity. In contrast, human and S. pombe Rap1s have just a single Myb motif and exhibit little or no DNAbinding activity. In addition, the apparent variations in the spacing between the Rap1 half sites in different organisms [e.g., 8 bp in S. cerevisiae and $7 \mathrm{bp}$ in C. albicans (center-to-center distance)] are consistent with adaptions involving altered dispositions between the two Myb motifs (Figure 5). With regard to the Cdc13 family members, the utilization of two sets of protein-DNA contacts for high affinity binding is not universal. While Candida Cdc13 dimers probably all require two sets of DBD-DNA interactions to bind stably to G-tails, S. cerevisiae $\mathrm{Cdc13}$ (despite forming dimers) binds G-tail with exceptionally high affinity using just one DBD-DNA interaction. This impressive feat of $\mathrm{ScCdc13}$ is accomplished by expanding the typical OB-DNA interface through the acquisition of an extended and structurally well-defined L23. That is, rather than adding a second set of protein-DNA interaction, ScCdc13 was able to drastically expand the first set to enhance binding affinity. S. castellii Cdc13, another 4-OB fold Cdc13, also appears to need just one DBD-DNA interaction for high affinity binding. Whether this property applies to other large Cdc13s (e.g., K. lactis Cdc13) is an interesting question for future investigation.

A special case of achieving high affinity binding through two sets of protein-DNA interactions, employed by members of Cdc13 family only (specifically CpCdc13A and $C p C \mathrm{Cdc} 13 \mathrm{~B}$ ), involves gene duplication and hetero-dimerization. Compared to homodimerization, this strategy has the advantage of allowing $\mathrm{Cdc} 13$ dimers to recognize a more complex target sequence made up of two distinct half sites. This advantage makes heterodimerization an especially adaptive strategy for the recognition of Candida telomere repeat units, which are long and complex.

The second common mechanistic feature that may enable ready adaption of Rap1 and Cdc13 to new telomere sequences is the ability of the DNA-binding surfaces of these proteins to undergo local conformational changes to accommodate different target sequence. This was implied by the huge number of Rap1 target sites in the $S$. cerevisiae genome and the very loose consensus sequence obtained for this protein. High resolution structural analyses of Rap1 bound to three target sequences provided amply illustration of this local flexibility at the molecular level (Taylor et al., 2000). In the case of Cdc13, there is no direct evidence yet for this local conformational flexibility. However, analysis of another ss telomere binding protein (TEBP from Oxytricha nova) revealed considerable tolerance of its binding surface to different sequences (Theobald and Schultz, 2003). Moreover, the intrinsically greater flexibility of ssDNA may further contribute to the ability of Cdc13 to accommodate sequence changes. An illustration of this, uncovered by investigation of onTEBP, is termed nucleotide shuffling, which involves the extrusion of a nucleotide away from the protein surface, and thus an alteration in the registry of the DNA (Theobald and Schultz, 2003). This phenomenon 
can conceivably allow insertional mutations in telomere DNA to be easily accommodated by Cdc13. Thus, both sequence-specific ss and dsDNA-binding proteins can exhibit limited versatility in binding multiple target sequences. Nevertheless, as noted earlier, promiscuous binding of telomere proteins to non-telomeric sites would probably be highly detrimental to cell physiology. Thus, limited versatility of Rap 1 and Cdc13 in sequence-specific

\section{References}

Anderson, E. M., Halsey, W. A., and Wuttke, D. S. (2003). Site-directed mutagenesis reveals the thermodynamic requirements for single-stranded DNA recognition by the telomere-binding protein Cdc13. Biochemistry 42, 3751-3758. doi: $10.1021 / \mathrm{bi027047c}$

Arat, N. O., and Griffith, J. D. (2012). Human Rap1 interacts directly with telomeric DNA and regulates TRF2 localization at the telomere. J. Biol. Chem. 287, 41583-41594. doi: 10.1074/jbc.M112.415984

Autexier, C., and Lue, N. F. (2006). The structure and function of telomerase reverse transcriptase. Annu. Rev. Biochem. 75, 493-517. doi: 10.1146/annurev. biochem.75.103004.142412

Blackburn, E. H., and Collins, K. (2011). Telomerase: an RNP enzyme synthesizes DNA. Cold Spring Harb. Perspect. Biol. 3, a003558. doi: 10.1101/cshperspect.a003558

Bochkarev, A., and Bochkareva, E. (2004). From RPA to BRCA2: lessons from single-stranded DNA binding by the OB-fold. Curr. Opin. Struct. Biol. 14, 36-42. doi: 10.1016/j.sbi.2004.01.001

Buchman, A., Lue, N., and Kornberg, R. (1988). Connections between transcriptional activators, silencers, and telomeres as revealed by functional analysis of a yeast DNA-binding protein. Mol. Cell. Biol. 8, 5086-5099.

Court, R., Chapman, L., Fairall, L., and Rhodes, D. (2005). How the human telomeric proteins TRF1 and TRF2 recognize telomeric DNA: a view from high-resolution crystal structures. EMBO Rep. 6, 39-45. doi: 10.1038/sj.embor. 7400314

de Lange, T. (2009). How telomeres solve the end-protection problem. Science 326, 948-952. doi: 10.1126/science.1170633

Dujon, B., Sherman, D., Fischer, G., Durrens, P., Casaregola, S., Lafontaine, I., et al. (2004). Genome evolution in yeasts. Nature 430, 35-44. doi: 10.1038/ nature 02579

Eldridge, A. M., Halsey, W. A., and Wuttke, D. S. (2006). Identification of the determinants for the specific recognition of single-strand telomeric DNA by Cdc13. Biochemistry 45, 871-879. doi: 10.1021/bi0512703

Eldridge, A. M., and Wuttke, D. S. (2008). Probing the mechanism of recognition of ssDNA by the Cdc13-DBD. Nucleic Acids Res. 36, 1624-1633. doi: 10.1093/nar/gkn017

Fairall, L., Chapman, L., Moss, H., de Lange, T., and Rhodes, D. (2001). Structure of the TRFH dimerization domain of the human telomeric proteins TRF1 and TRF2. Mol. Cell 8, 351-361. doi: 10.1016/S1097-2765(01)00321-5

Feeser, E. A., and Wolberger, C. (2008). Structural and functional studies of the Rap1 C-terminus reveal novel separation-of-function mutants. J. Mol. Biol. 380, 520-531. doi: 10.1016/j.jmb.2008.04.078

Garvik, B., Carson, M., and Hartwell, L. (1995). Single-stranded DNA arising at telomeres in cdc13 mutants may constitute a specific signal for the RAD9 checkpoint. Mol. Cell. Biol. 15, 6128-6138.

Giraldo, R., and Rhodes, D. (1994). The yeast telomere-binding protein RAP1 binds to and promotes the formation of DNA quadruplexes in telomeric DNA. EMBO J. 13, 2411-2420.

Giraud-Panis, M. J., Teixeira, M. T., Geli, V., and Gilson, E. (2010). CST meets shelterin to keep telomeres in check. Mol. Cell 39, 665-676. doi: 10.1016/ j.molcel.2010.08.024

Hedges, S. B. (2002). The origin and evolution of model organisms. Nat. Rev. Genet. 3, 838-849. doi: 10.1038/nrg929

Hsu, C. L., Chen, Y. S., Tsai, S. Y., Tu, P. J., Wang, M. J., and Lin, J. J. (2004). Interaction of Saccharomyces Cdc13p with Pol1p, Imp4p, Sir4p and Zds2p is involved in telomere replication, telomere maintenance and cell growth control. Nucleic Acids Res. 32, 511-521. doi: 10.1093/nar/gkh203 recognition probably is reflective of a finely calibrated evolutionary compromise.

\section{Acknowledgment}

Works in the authors' laboratories are supported by National Science Foundation (MCB-1157305) and NIH (GM107287).

Huet, J., Cottrelle, P., Cool, M., Vignais, M. L., Thiele, D., Marck, C., et al. (1985) A general upstream binding factor for genes of the yeast translational apparatus. EMBO J. 4, 3539-3547.

Hughes, T. R., Weilbaecher, R. G., Walterscheid, M., and Lundblad, V. (2000). Identification of the single-strand telomeric DNA binding domain of the Saccharomyces cerevisiae Cdc13 protein. Proc. Natl. Acad. Sci. U.S.A. 97, 6457-6462. doi: 10.1073/pnas.97.12.6457

Idrissi, F. Z., Fernandez-Larrea, J. B., and Pina, B. (1998). Structural and functional heterogeneity of Raplp complexes with telomeric and UASrpglike DNA sequences. J. Mol. Biol. 284, 925-935. doi: 10.1006/jmbi.1998. 2215

Idrissi, F. Z., and Pina, B. (1999). Functional divergence between the half-sites of the DNA-binding sequence for the yeast transcriptional regulator Rap1p. Biochem. J. 341(Pt 3), 477-482. doi: 10.1042/0264-6021:3410477

Jain, D., and Cooper, J. P. (2011). Telomeric strategies: means to an end. Annu. Rev. Genet. 44, 243-269. doi: 10.1146/annurev-genet-102108-134841

Kabir, S., Hockemeyer, D., and de Lange, T. (2014). TALEN gene knockouts reveal no requirement for the conserved human shelterin protein Rap1 in telomere protection and length regulation. Cell Rep. 9, 1273-1280. doi: 10.1016/j.celrep.2014.10.014

Kanoh, J., and Ishikawa, F. (2001). spRap1 and spRif1, recruited to telomeres by Taz1, are essential for telomere function in fission yeast. Curr. Biol. 11, 1624-1630. doi: 10.1016/S0960-9822(01)00503-6

Konig, P., Giraldo, R., Chapman, L., and Rhodes, D. (1996). The crystal structure of the DNA-binding domain of yeast RAP1 in complex with telomeric DNA. Cell 85, 125-136. doi: 10.1016/S0092-8674(00)81088-0

Lansdorp, P. M. (2005). Major cutbacks at chromosome ends. Trends Biochem. Sci. 30, 388-395. doi: 10.1016/j.tibs.2005.05.004

Lavoie, H., Hogues, H., Mallick, J., Sellam, A., Nantel, A., and Whiteway, M. (2010). Evolutionary tinkering with conserved components of a transcriptional regulatory network. PLoS Biol. 8:e1000329. doi: 10.1371/journal.pbio.1000329

Lei, M., Podell, E. R., Baumann, P., and Cech, T. R. (2003). DNA self-recognition in the structure of Pot1 bound to telomeric single-stranded DNA. Nature 426, 198-203. doi: 10.1038/nature02092

Lei, M., Podell, E. R., and Cech, T. R. (2004). Structure of human POT1 bound to telomeric single-stranded DNA provides a model for chromosome endprotection. Nat. Struct. Mol. Biol. 11, 1223-1229. doi: 10.1038/nsmb867

Li, B., Oestreich, S., and de Lange, T. (2000). Identification of human Rap1: implications for telomere evolution. Cell 101, 471-483. doi: 10.1016/S00928674(00)80858-2

Lieb, J. D., Liu, X., Botstein, D., and Brown, P. O. (2001). Promoter-specific binding of Rap1 revealed by genome-wide maps of protein-DNA association. Nat. Genet. 28, 327-334. doi: 10.1038/ng569

Lopez, M. C., Smerage, J. B., and Baker, H. V. (1998). Multiple domains of repressor activator protein 1 contribute to facilitated binding of glycolysis regulatory protein 1. Proc. Natl. Acad. Sci. U.S.A. 95, 14112-14117. doi: 10.1073/ pnas.95.24.14112

Lue, N. F. (2010). Plasticity of telomere maintenance mechanisms in yeast. Trends Biochem. Sci. 35, 8-17. doi: 10.1016/j.tibs.2009.08.006

Lue, N. F., and Chan, J. (2013). Duplication and functional specialization of the telomere-capping protein Cdc13 in Candida species. J. Biol. Chem. 288, 29115-29123. doi: 10.1074/jbc.M113.506519

Lue, N. F., Chan, J., Wright, W. E., and Hurwitz, J. (2014). The CDC13-STN1-TEN1 complex stimulates Pol alpha activity by promoting RNA priming and primaseto-polymerase switch. Nat. Commun. 5, 5762. doi: 10.1038/ncomms6762

Lustig, A. (2003). Clues to catastrophic telomere loss in mammals from yeast telomere rapid deletion. Nat. Rev. Genet. 4, 916-923. doi: 10.1038/nrg1207 
Martinez, P., Thanasoula, M., Carlos, A. R., Gomez-Lopez, G., Tejera, A. M., Schoeftner, S., et al. (2010). Mammalian Rapl controls telomere function and gene expression through binding to telomeric and extratelomeric sites. Nat. Cell Biol. 12, 768-780. doi: 10.1038/ncb2081

Mason, M., Wanat, J. J., Harper, S., Schultz, D. C., Speicher, D. W., Johnson, F. B., et al. (2012). Cdc13 OB2 dimerization required for productive Stn1 binding and efficient telomere maintenance. Structure 21, 109-120. doi: 10.1016/j.str.2012.10.012

Mitchell, M. T., Smith, J. S., Mason, M., Harper, S., Speicher, D. W., Johnson, F. B., et al. (2010). Cdc13 N-terminal dimerization, DNA binding, and telomere length regulation. Mol. Cell. Biol. 30, 5325-5334. doi: 10.1128/MCB. 00515-10

Mitton-Fry, R., Anderson, E., Hughes, T., Lundblad, V., and Wuttke, D. (2002). Conserved structure for single-stranded telomeric DNA recognition. Science 296, 145-147. doi: 10.1126/science.1068799

Mitton-Fry, R. M., Anderson, E. M., Theobald, D. L., Glustrom, L. W., and Wuttke, D. S. (2004). Structural basis for telomeric single-stranded DNA recognition by yeast Cdc13. J. Mol. Biol. 338, 241-255. doi: 10.1016/j.jmb.2004.01.063

Miyake, Y., Nakamura, M., Nabetani, A., Shimamura, S., Tamura, M., Yonehara, S., et al. (2009). RPA-like mammalian Ctc1-Stn1-Ten1 complex binds to singlestranded DNA and protects telomeres independently of the Pot1 pathway. Mol. Cell 36, 193-206. doi: 10.1016/j.molcel.2009.08.009

Nandakumar, J., and Cech, T. R. (2013). Finding the end: recruitment of telomerase to telomeres. Nat. Rev. Mol. Cell Biol. 14, 69-82. doi: 10.1038/nrm3505

Nugent, C. I., Hughes, T. R., Lue, N. F., and Lundblad, V. (1996). Cdc13p: a single-strand telomeric DNA-binding protein with a dual role in yeast telomere maintenance. Science 274, 249-252. doi: 10.1126/science.274.5285.249

Olovnikov, A. M. (1996). Telomeres, telomerase, and aging: origin of the theory. Exp. Gerontol. 31, 443-448. doi: 10.1016/0531-5565(96)00005-8

O'Sullivan, R. J., and Karlseder, J. (2010). Telomeres: protecting chromosomes against genome instability. Nat. Rev. Mol. Cell Biol. 11, 171-181. doi: 10.1038/ nrm 2848

Pennock, E., Buckley, K., and Lundblad, V. (2001). Cdc13 delivers separate complexes to the telomere for end protection and replication. Cell 104, 387-396. doi: 10.1016/S0092-8674(01)00226-4

Pesole, G., Lotti, M., Alberghina, L., and Saccone, C. (1995). Evolutionary origin of nonuniversal CUGSer codon in some Candida species as inferred from a molecular phylogeny. Genetics 141, 903-907.

Pfeiffer, V., and Lingner, J. (2013). Replication of telomeres and the regulation of telomerase. Cold Spring Harb. Perspect. Biol. 5, a010405. doi: 10.1101/cshperspect.a010405

Pina, B., Fernandez-Larrea, J., Garcia-Reyero, N., and Idrissi, F. Z. (2003). The different (sur)faces of Raplp. Mol. Genet. Genomics 268, 791-798. doi: 10.1007/ s00438-002-0801-3

Podlevsky, J. D., Bley, C. J., Omana, R. V., Qi, X., and Chen, J. J. (2008). The telomerase database. Nucleic Acids Res. 36, D339-D343. doi: 10.1093/nar/gkm700

Price, C. M., Boltz, K. A., Chaiken, M. F., Stewart, J. A., Beilstein, M. A., and Shippen, D. E. (2010). Evolution of CST function in telomere maintenance. Cell Cycle 9, 3157-3165. doi: 10.4161/cc.9.16.12547

Qi, H., and Zakian, V. A. (2000). The Saccharomyces telomere-binding protein Cdc13p interacts with both the catalytic subunit of DNA polymerase $\alpha$ and the telomerase-associated Est1 protein. Genes Dev. 14, 1777-1788.

Rhee, H. S., and Pugh, B. F. (2011). Comprehensive genome-wide protein-DNA interactions detected at single-nucleotide resolution. Cell 147, 1408-1419. doi: 10.1016/j.cell.2011.11.013

Rhodin Edso, J., Gustafsson, C., and Cohn, M. (2011). Single- and double-stranded DNA binding proteins act in concert to conserve a telomeric DNA core sequence. Genome Integr. 2, 2. doi: 10.1186/2041-9414-2-2

Rhodin Edso, J., Tati, R., and Cohn, M. (2008). Highly sequence-specific binding is retained within the DNA-binding domain of the Saccharomyces castellii Cdc13 telomere-binding protein. FEMS Yeast Res. 8, 1289-1302. doi: 10.1111/j.15671364.2008.00431.x

Schwabe, J. W., Chapman, L., and Rhodes, D. (1995). The oestrogen receptor recognizes an imperfectly palindromic response element through an alternative side-chain conformation. Structure 3, 201-213. doi: 10.1016/S0969-2126(01) 00150-2
Sfeir, A., Kabir, S., Van Overbeek, M., Celli, G. B., and de Lange, T. (2010). Loss of Rap1 induces telomere recombination in the absence of NHEJ or a DNA damage signal. Science 327, 1657-1661. doi: 10.1126/science.1185100

Shore, D. (1994). RAP1: a protean regulator in yeast. Trends Genet. 10, 408-412. doi: 10.1016/0168-9525(94)90058-2

Shore, D., Stillman, D. J., Brand, A. H., and Nasmyth, K. A. (1987). Identification of silencer binding proteins from yeast: possible roles in SIR control and DNA replication. EMBO J. 6, 461-467.

Steinberg-Neifach, O., Wellington, K., Vazquez, L., and Lue, N. F. (2015). Combinatorial recognition of a complex telomere repeat sequence by the Candida parapsilosis Cdc13AB heterodimer. Nucleic Acids Res. 43, 2164-2176. doi: 10.1093/nar/gkv092

Stewart, J. A., Wang, F., Chaiken, M. F., Kasbek, C., Chastain, P. D. II, Wright, W. E., et al. (2012). Human CST promotes telomere duplex replication and general replication restart after fork stalling. EMBO J. 31, 3537-3549. doi: 10.1038/emboj.2012.215

Sun, J., Yang, Y., Wan, K., Mao, N., Yu, T. Y., Lin, Y. C., et al. (2011). Structural bases of dimerization of yeast telomere protein $\mathrm{Cdc} 13$ and its interaction with the catalytic subunit of DNA polymerase alpha. Cell Res. 21, 258-274. doi: $10.1038 / \mathrm{cr} .2010 .138$

Surovtseva, Y. V., Churikov, D., Boltz, K. A., Song, X., Lamb, J. C., Warrington, R., et al. (2009). Conserved telomere maintenance component 1 interacts with STN1 and maintains chromosome ends in higher eukaryotes. Mol. Cell 36, 207-218. doi: 10.1016/j.molcel.2009.09.017

Taylor, H. O., O’Reilly, M., Leslie, A. G., and Rhodes, D. (2000). How the multifunctional yeast Raplp discriminates between DNA target sites: a crystallographic analysis. J. Mol. Biol. 303, 693-707. doi: 10.1006/jmbi.2000.4161

Theobald, D. L., Mitton-Fry, R. M., and Wuttke, D. S. (2003). Nucleic acid recognition by OB-fold proteins. Annu. Rev. Biophys. Biomol. Struct. 32, 115-133. doi: 10.1146/annurev.biophys.32.110601.142506

Theobald, D. L., and Schultz, S. C. (2003). Nucleotide shuffling and ssDNA recognition in Oxytricha nova telomere end-binding protein complexes. EMBO J. 22, 4314-4324. doi: 10.1093/emboj/cdg415

Vignais, M. L., Huet, J., Buhler, J. M., and Sentenac, A. (1990). Contacts between the factor TUF and RPG sequences. J. Biol. Chem. 265, 14669-14674.

Wahlin, J., and Cohn, M. (2000). Saccharomyces cerevisiae RAP1 binds to telomeric sequences with spatial flexibility. Nucleic Acids Res. 28, 2292-2301. doi: $10.1093 /$ nar/28.12.2292

Wahlin, J., and Cohn, M. (2002). Analysis of the RAP1 protein binding to homogeneous telomeric repeats in Saccharomyces castellii. Yeast 19, 241-256. doi: 10.1002/yea.816

Wu, Y., and Zakian, V. A. (2011). The telomeric Cdc13 protein interacts directly with the telomerase subunit Est1 to bring it to telomeric DNA ends in vitro. Proc. Natl. Acad. Sci. U.S.A. 108, 20362-20369. doi: 10.1073/pnas.1100281108

Yarragudi, A., Parfrey, L. W., and Morse, R. H. (2007). Genome-wide analysis of transcriptional dependence and probable target sites for Abf1 and Rap1 in Saccharomyces cerevisiae. Nucleic Acids Res. 35, 193-202. doi: 10.1093/nar/gkl1059

Yu, E. Y., Sun, J., Lei, M., and Lue, N. F. (2012). Analyses of Candida Cdc13 orthologues revealed a novel OB fold dimer arrangement, dimerization-assisted DNA binding, and substantial structural differences between Cdc13 and RPA70. Mol. Cell. Biol. 32, 186-198. doi: 10.1128/MCB.05875-11

Yu, E. Y., Yen, W. F., Steinberg-Neifach, O., and Lue, N. F. (2010). Rap1 in Candida albicans: an unusual structural organization and a critical function in suppressing telomere recombination. Mol. Cell. Biol. 30, 1254-1268. doi: 10.1128/ MCB.00986-09

Conflict of Interest Statement: The authors declare that the research was conducted in the absence of any commercial or financial relationships that could be construed as a potential conflict of interest.

Copyright (c) 2015 Steinberg-Neifach and Lue. This is an open-access article distributed under the terms of the Creative Commons Attribution License (CC BY). The use, distribution or reproduction in other forums is permitted, provided the original author(s) or licensor are credited and that the original publication in this journal is cited, in accordance with accepted academic practice. No use, distribution or reproduction is permitted which does not comply with these terms. 